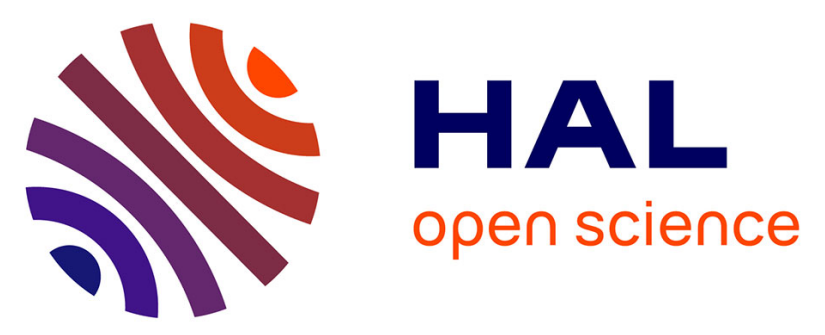

\title{
Proton exchange membrane fuel cell remaining useful life prognostics considering degradation recovery phenomena
}

Dacheng Zhang, Catherine Cadet, Nadia Yousfi-Steiner, Christophe Bérenguer

\section{- To cite this version:}

Dacheng Zhang, Catherine Cadet, Nadia Yousfi-Steiner, Christophe Bérenguer. Proton exchange membrane fuel cell remaining useful life prognostics considering degradation recovery phenomena. Proceedings of the Institution of Mechanical Engineers, Part O: Journal of Risk and Reliability, 2018, 232 (4), pp.415 - 424. 10.1177/1748006X18776825 . hal-01885664

\section{HAL Id: hal-01885664 \\ https://hal.univ-grenoble-alpes.fr/hal-01885664}

Submitted on 8 Jun 2021

HAL is a multi-disciplinary open access archive for the deposit and dissemination of scientific research documents, whether they are published or not. The documents may come from teaching and research institutions in France or abroad, or from public or private research centers.
L'archive ouverte pluridisciplinaire HAL, est destinée au dépôt et à la diffusion de documents scientifiques de niveau recherche, publiés ou non, émanant des établissements d'enseignement et de recherche français ou étrangers, des laboratoires publics ou privés. 


\title{
Proton exchange membrane fuel cell remaining useful life prognostics considering degradation recovery phenomena
}

\section{Dacheng Zhang ${ }^{1}$, Catherine Cadet ${ }^{1}$, Nadia Yousfi-Steiner ${ }^{2,3,4}$ and Christophe Bérenguer $^{1}$}

\begin{abstract}
The present work explores the challenges of handling the recovery phenomena in the degradation behavior of the Proton Exchange Membrane Fuel Cells, from the perspective of the prognostics. An adaptive Prognostics and Health Management approach with additional knowledge, such as the Electrochemical Impedance Spectroscopy, from the State of Health characterization, is applied on two fuel cell stacks under both stationary and quasi-dynamic operating regimes. Some improvements in the prognostic performance are obtained in the view of the Remaining Useful Life predictions by comparing with a classical Particle Filtering-based prognostic approach.
\end{abstract}

\section{Keywords}

Proton Exchange Membrane Fuel Cell (PEMFC), Prognostics and Health Management (PHM), Electrochemical Impedance Spectroscopy (EIS), Remaining Useful Life (RUL), Particle Filtering (PF)

\section{Introduction}

In mobile applications, the spotlight is presently on the fuel cell systems among energy conversion devices. Proton Exchange Membrane Fuel Cell (PEMFC) has been considered as one of the most

\footnotetext{
${ }^{1}$ Univ. Grenoble Alpes, CNRS, Grenoble INP, GIPSA-Lab, 38000 Grenoble, France

${ }^{2}$ FEMTO-ST, CNRS / Univ. Bourgogne Franche-Comte, Belfort, France

${ }^{3}$ FCLAB, CNRS / Univ. Bourgogne Franche-Comte, Belfort, France

${ }^{4}$ Labex ACTION, CNRS, Belfort, France

Corresponding author:

Christophe Bérenguer, GIPSA-Lab, 11 rue des Mathématiques, Grenoble Campus BP46, 38402 SAINT MARTIN D'HERES CEDEX, FRANCE

Email: christophe.berenguer@gipsa-lab.grenoble-inp.fr
} 
promising technologies for both stationary and mobile applications among different types of fuel cells. Like other kinds of fuel cells, the PEMFC is a device which generates electricity from electrochemical reactions continuously for as long as the inputs are supplied and it produces eco-friendly by-products (water and heat):

$$
\begin{aligned}
\text { Anode }: & \mathrm{H}_{2} \rightarrow 2 \mathrm{H}^{+}+2 e^{-} \\
\text {Cathode }: & \frac{1}{2} \mathrm{O}_{2}+2 \mathrm{H}^{+}+2 e^{-} \rightarrow \mathrm{H}_{2} \mathrm{O} \\
\text { Overall }: & \frac{1}{2} \mathrm{O}_{2}+\mathrm{H}_{2} \rightarrow \mathrm{H}_{2} \mathrm{O}
\end{aligned}
$$

It is a complex system with dynamic conditions and local heterogeneities. The main parts of the cell are the bipolar plates, the gas diffusion layer that transports gases and excess of water, the electrodes where half-reactions take place, and the membrane which transports $H^{+}$and water. The membrane should be well hydrated to ensure a good $\mathrm{H}^{+}$conductance.

Even if this technology is close to being competitive, it is not yet ready to be considered for a large scale industrial deployment because of its limited durability. The Prognostics and Health Management (PHM) approaches are considered as favorable solutions to manage PEMFC's reliability and durability. The PHM is a combination of several processes to monitor, analyze and master the systems' State of Health (SOH). The prognostic results can be helpful in making decisions such as maintenance scheduling and control strategy, such that early notification of degradation can be detected and early failures can be avoided. Meanwhile, the repairing cost can be reduced. Prognostic techniques can provide an estimation of a fuel cell's degradation state and a prediction for its Remaining Useful Life (RUL). It can thus help to better manage the health and residual life of the fuel cell system and to improve its performance, for example through the control of the operating conditions or by adapted maintenance. The durability of PEMFC can be affected by both internal and external aging factors. The external aging factors are calendar aging under constant optimal conditions; start/stop cycles and deficient operating conditions such as temperature, pressure and poor water management. The consequences are materials degradation that can be seen in main fuel cell components (Dubau et al. 2014). Mechanical, thermal, chemical and electrochemical mechanisms could lead to the change of physical properties and even damage the stack. Those degradations of the PEMFC's stacks cannot be directly measured. For RUL determination, the output power degradation is mostly used as an aging indicator. However, the power degradation only reflects a combination of the impacts of all aging factors. Hence, different behaviors can be observed from the same stack under the same operating conditions, and identical stacks (different stacks of the same type and manufacture) under the same conditions can also behave differently. To characterize the State of Health (SOH) of a PEMFC stack, the Electrochemical Impedance Spectroscopy (EIS) and polarization measurements are applied at regular time intervals. These measurements also induce a particular behavior of PEMFC which leads to sudden drops and jumps of output power and which can be categorized into recovery phenomena. All the aforementioned factors bring difficulties and challenges into PEMFC prognostics.

A sequence of works has been conducted in this field of PEMFC prognostics, following different approaches: model-based or data-based, even if the proposed approaches are often hybrid, in the sense that they use both models and data. The state-of-the-art and remaining challenges of PHM for PEMFC have been reviewed in (Jouin et al. 2013), which identifies the crucial requirements of a vast quantity 
of data to develop complete models for behavior, aging, and degradation. Moreover, data for different applications and different operating conditions should be gathered to ensure the generality and the transferability of the developed approaches. Approaches based on machine learning techniques have been proposed: the method in (Morando et al. 2017) requires a large data series and a long time for tuning of several model parameters. The work in (Javed et al. 2016) has overcome the data limitation, but it needs state constraints to guarantee the RUL predictions. For approaches involving a model, the modeling level depends on the level of understanding of PEMFC behaviors. For some not-well-understood behaviors, e.g. the recovery phenomena, it can be interesting to rely on empirical (based on experimental data) modeling of degradation trends to improve the precision of final prediction. When feasible, a physicsbased behavioral model to reproduce PEMFC aging behavior can be proposed, as in (Lechartier et al. 2015). The model is composed of a static part and dynamic parts that are independent. The static part is developed thanks to equations describing the physical phenomena and is based on the Butler-Volmer law that takes into account the activation loss at the cathode and the anode. In (Bressel et al. 2016), the PEMFC RUL prediction subjected to a $\mu$-CHP profile is carried out by using an Extended Kalman Filter (EKF) throughout a physics-based voltage model.

For the problem of PEMFC RUL prediction, taking into account the uncertainty is also mandatory. It has been proven that the Bayesian estimation techniques provide a framework which can deal with high uncertainties in degradation processes (Vachtsevanos et al. 2006). Bayesian estimation with particle filters is not limited by either linearity or Gaussian noise assumption. Approaches based on particle filters (PF) are more and more employed for prognostics purposes (Jouin et al. 2016b) and are chosen for the degradation path estimation and prediction in this study. In (Jha et al. 2016), the authors proposed parameters estimated from the PEMFC polarization curves as $\mathrm{SOH}$ degradation indicators. Their method gives promising results despite its complexity and shows that the SOH characterization of PEMFC can be very helpful in RUL prediction. In (Kimotho et al. 2014), the authors estimate the RUL by modeling and tracking the voltage degradation trend using a Particle Filtering (PF) framework. They introduced a self-healing factor after each characterization and adapted the degradation model parameters to fit the changing degradation trend. In (Jouin et al. 2016a), the authors propose an empirical model for power degradation taking into account recovery phenomena based on different features extracted from the degradation pattern.

These works provide a promising basis for PEMFC RUL prediction based on degradation trend. It has to be noticed however that, in those works, the output power (or voltage) of the stack is used as the only indicator of PEMFC degradation. As explained in detail in the next section, our objective in this work is to use the power degradation trend in combination with parameters estimated from another $\mathrm{SOH}$ characterization - the Electrochemical Impedance Spectroscopy (EIS), which has been shortly addressed in (Zhang et al. 2016).

The remainder of this paper is organized as follows. In the first section, the problem statement and the motivation for the work contribution are introduced. Then, the PF-based prognostic algorithm enabling the RUL prediction and the criteria of prediction quality evaluation are described in the second section. The third section presents the proposed approach aiming at integrating information from the stack characterization in the prognostic procedure. Finally, the experimental results are proposed to illustrate the performance and interest of the proposed method. 


\section{Problem Statement and Work Objectives}

The stack voltage drop or power drop is the most obvious deterioration indicator used to implement a procedure of deterioration tracking and prognostics. However, it might not be the best approach because of at least two reasons: i) the voltage drop can be seen as a superficial symptom of the deeper deterioration of the stack, and might not characterize it completely; ii) some recovery phenomena can appear on the stack voltage evolution after e.g. a characterization procedure is carried out on the stack or after a reversible fault disappearance. Our objective in this work is to investigate how information on the deeper characterization of the stack can be used to better take into account the recovery phenomena and to improve the prognostic performance.

\section{Stack Degradation}

The voltage drop is commonly used to indicate stack degradation in time. Due to degradation phenomena, the ability of a fuel cell to keep a sufficient power will fall. In this paper, two long-term experimental data sets from two identical PEMFC stacks, referred to as FC1 and FC2, will be used. These data are taken from (Gouriveau et al. 2014).

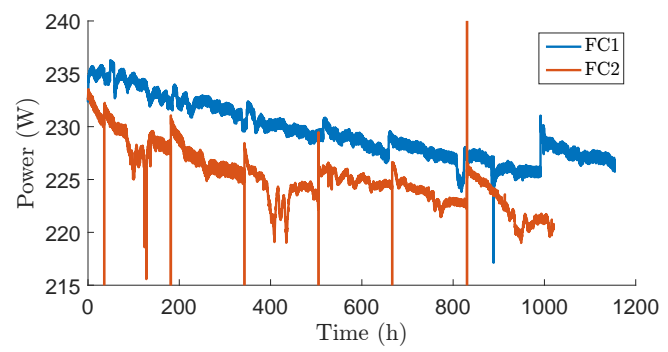

Figure 1. Power degradation in PEMFC stacks.

Figure 1 shows the stacks' power signals (Gouriveau et al. 2014) over time under both nominal and dynamic current operating conditions. FC1 is operated under the nominal condition, and FC2 is operated under dynamic current of $10 \%$ oscillations at a high frequency of $5 \mathrm{kHz}$. Degradation indicators are supposed to only decrease with time. However, in this figure, one can see that some increases are also noticeable. Thus, several peaks at certain time instances can be observed which are the consequence of the characterization measurements which take place each week. These measures lead to sudden power peaks which are due to temporary recovery phenomena in the stack. Furthermore, the general shape of FC2 is different from the FC1, and dramatic drops occur. These drops are due to unknown reversible phenomena as the power recovers its previous level after some period.

Thus the prognostic algorithm has to face a specific difficulty i.e. the power trend is not only a degradation indicator but also includes reversible phenomena. However, the irreversible degradation of the PEMFC stack can be assessed based on the internal characterizations of the stack's State of Health (SOH), such as the Electrochemical Impedance Spectroscopy (EIS). 


\section{Stack Characterization}

The Electrochemical Impedance Spectroscopy (EIS) is commonly used to characterize different phenomena and component materials inside the stack. For a given frequency range, the internal impedance allows constructing an EIS which is represented by the Nyquist diagram. The time impact is depicted in the EIS in Figure 2.

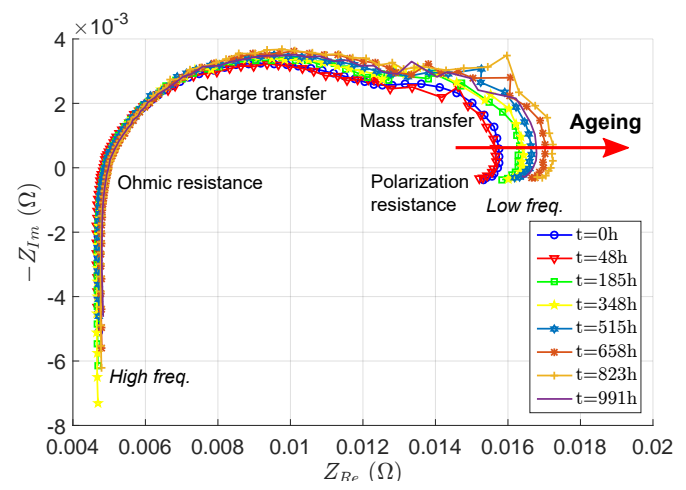

Figure 2. EIS diagram of a PEMFC.

Different phenomena can be directly observed from the shape of the EIS curve:

- An inductive part at the highest frequencies due to connection cables inductance.

- A capacitive loop due to the accumulation of charges at the electrode-electrolyte interface and the resistance of transferring electrons.

- A diffusional part at lowest frequencies due to diffusion of species (reactants and products). It is dominated by the electrochemical reaction with the slowest kinetics.

Parameters such as resistance, capacitance, and inductance can be identified from EIS by an Equivalent Circuit Model (ECM) by fitting the ECM to the EIS curves.

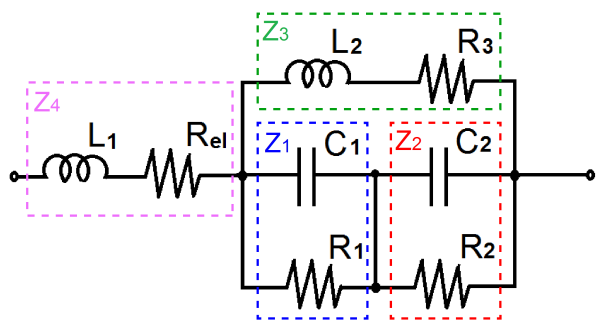

Figure 3. Equivalent circuit model (ECM) of a PEMFC cell.

Figure 3 shows an ECM proposed by (Kim et al. 2015). The equivalent impedances of the PEMFC is described as: 


$$
Z_{e q}=\frac{\left(Z_{1}+Z_{2}\right) \cdot Z_{3}}{Z_{1}+Z_{2}+Z_{3}}+Z_{4}
$$

The aging effect can be thus investigated by the changes of the impedance. The work presented in this paper precisely aims at using this stack aging information synthesized from the stack characterization results within the prognostic procedure.

\section{Using Stack Characterization Information for Prognostics}

The RUL prediction is often based only on one source of deterioration and most of the time the existence of multiple types of deterioration in decision making is ignored. Therefore, this work aims to integrate additional information (i.e. EIS characterization) to improve the RUL estimation accuracy based on the stack voltage or power degradation. The original contribution of this approach does not really and only lie in the integration of the recovery phenomena since this issue has also been considered in other works (Kimotho et al. 2014; Jouin et al. 2016a). One of the difficulty is that the recovery phenomena and their amplitude varies with time (as shown by experimental evidence, for example in Figure 4); the same model for the recovery phenomena cannot be used over the entire life of the PEMFC stack. If we consider the same issue of prognostics with time-varying recovery phenomena as other authors did, we do not resort to the same solution. Jouin et al. (2016a) consider a model with time-dependent parameters for the recovery phenomena whereas we consider in this work a model whose parameters are function of the internal state of the stack (internal impedance). The solution in (Jouin et al. 2016a) relies on the use of joint multi-stage particle filters to track both the power decrease itself and the time evolution of the coefficients of the power deterioration model with the recovery phenomena. Our contribution consists in using the information delivered by the EIS characterization to update the model parameters of the recovery phenomena. Our original proposition is hence to use information gathered at different levels in the stack (i.e. power, but also EIS based SOH characterization summarized by an equivalent impedance) to better adapt the deterioration estimation to its true state and to better predict the RUL. The two next sections develop this approach.

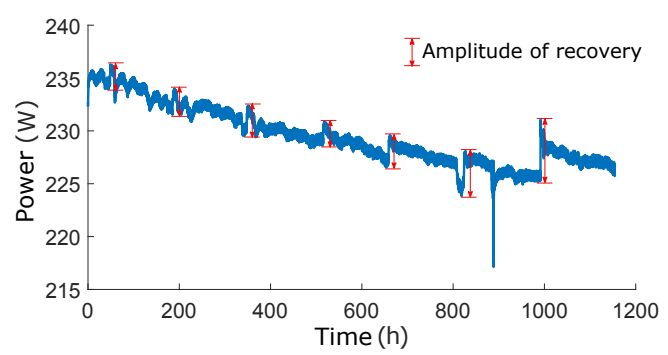

Figure 4. Recovery jumps and their variable amplitude over the stack lifetime 


\section{Particle Filtering-based Prognostics}

Particle Filtering (PF) is a hybrid (i.e. data- and model-based) method whose application in prognostics aims at tracking the evolution of an equipment degradation state from a sequence of noisy measurements; it relies on state-space description of the system evolution and observation with possibly non-linear and non-Gaussian features, and it resorts to Bayesian algorithms (Arulampalam et al. 2002). The RUL of the equipment is defined as the residual life from prediction time before the End of Life (EOL) when the degradation state reaches a preset Failure Threshold (FT). From a given prediction point in time $t_{p}$, the RUL can be predicted by propagating the estimated state through the degradation evolution, as illustrated in Figure 5.

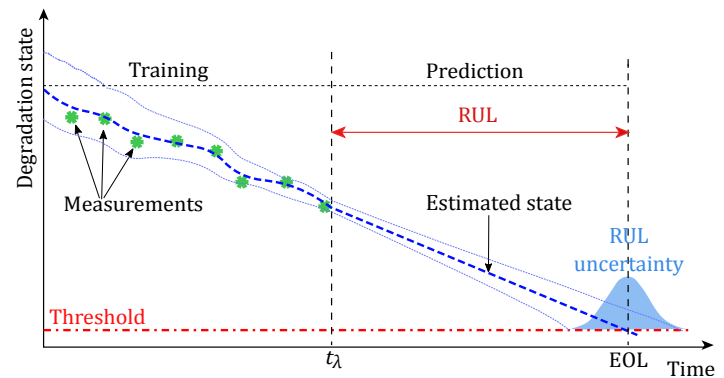

Figure 5. PF-based prognostics for RUL prediction

\section{Particle Filtering Algorithm}

Consider a Markovian system under operation subject to degradation from new till its EOL. The deterioration dynamics and its observation are assumed to be governed by a discrete-time state transition model:

$$
\begin{gathered}
x_{k}=f_{k}\left(x_{k-1}, \omega_{k-1}, \Theta_{k-1}\right) \\
z_{k}=h_{k}\left(x_{k}, \nu_{k}\right)
\end{gathered}
$$

where $k$ is the time index, $x$ is the system deterioration state, $z$ is the measurement, $f$ is the degradation model (state transition function), $\omega$ is the system noise, $\Theta$ is the vector of the model parameters $\left(\Theta=\left[\theta_{1}, \theta_{2}, \ldots\right]\right), h$ is the measurement model and $\nu$ is the measurement noise. Both the process noise $\omega_{k}$ and the observation noise $\nu_{k}$ are assumed to be sampled from a zero-mean Gaussian distribution, i.e. $\omega_{k} \sim \mathcal{N}\left(0, \sigma_{\omega k}^{2}\right)$ and $\nu_{k} \sim \mathcal{N}\left(0, \sigma_{\nu k}^{2}\right)$. In a PF framework (Arulampalam et al. 2002), the approximation of probability distribution of the deterioration state and the estimation of the deterioration state itself is based on the generated particles and their associated weights. The Bayesian update is processed sequentially by propagating particles carrying probabilistic information on the unknown states and model parameters and consists in 3 mains steps i) particles generation \& propagation; ii) weights estimation and iii) resampling, as summarized in Algorithm 1. The probabilistic model for the particles propagation relies on the state transition model (3a) and the probability distribution of the process noise $\omega_{k}$. The main steps are as follows: 
1. Propagate $i=1, \ldots, n$ particles representing the system state probability density function (PDF) from $x_{k-1}$ to $x_{k}$ by state transition model described in Equation (3a) (line 5).

2. For each particle, estimate the associated weight by calculating its likelihood of $x_{k}^{i}$ given an online measurement $z_{k}$, which quantifies the degree of matching between the particle and the online measurement (line 6). This gives the corresponding weight of each particle (assuming the measurement noise $\nu_{k} \sim \mathcal{N}\left(0, \sigma_{\nu k}^{2}\right)$ is normally distributed):

$$
\mathcal{L}\left(z_{k} \mid x_{k}^{i}, \sigma_{\nu k}^{i}\right)=\frac{1}{\sqrt{2 \pi} \sigma_{\nu k}^{i}} \exp \left[-\frac{1}{2}\left(\frac{z_{k}-x_{k}^{i}}{\sigma_{\nu k}^{i}}\right)^{2}\right]
$$

3. Re-sampling ( $\mathrm{Li}$ et al. 2015) to remove the particles with small weights compared to a chosen weight limit, the ones with great weights are duplicated which represent the estimated posterior PDF (line 10 to 17).

4. The posterior PDF built using resampling in step (3) is used as the prior back into step (1). This is performed until the online measurement is no longer available (prediction time $t_{\lambda}=k_{p} \cdot \Delta t$ reached).

For the RUL prediction (Algorithm 2), the posterior PDF of the state and model parameters, given the observation sequence up to time $t_{p}$, are used to estimate the future evolution of the particles. The RUL PDF can be obtained when the particles of system state reach the preset failure threshold by extrapolating the estimated degradation evolution.

\section{PF Algorithm with Recovery Phenomena}

The algorithm is then adapted to take into account the EIS and polarization measurements, which occur at characterization time step $C=\left[c_{1}, c_{2}, \ldots\right]$. At these time steps, the degradation model Equation (3a) is then replaced by a model including recovery phenomena $\left(x_{c}\right)$. The Algorithm 1 is modified such that line 5 is replaced by Algorithm 3. In the case where the EIS and polarization measurement times are known after $t_{p}$, Algorithm 3 can also be modified to take this into account.

\section{Performance Evaluation}

To evaluate the quality of the performance of predicted RULs, the prognostic metrics proposed by (Saxena et al. 2010) and (Rigamonti et al. 2016) are applied.

Accuracy The accuracy $A c c_{\lambda}$ is computed from the RUL prediction error relative to the true RUL:

$$
A c c_{\lambda}=1-\frac{\left|R U L_{\lambda}^{*}-\widehat{R U L}_{\lambda}\right|}{R U L_{\lambda}^{*}}
$$

where $R U L_{\lambda}^{*}$ the ground true RUL and $\widehat{R U L}_{\lambda}$ the median value of predicted RULs at prediction time $t_{\lambda}$. Larger values of $A c c_{\lambda}$ indicate better accuracy.

$\alpha-\lambda$ Accuracy The $\alpha-\lambda$ metric is a binary index which considers whether the predicted RUL at time $t_{\lambda}$ lies within the $\pm \alpha$ interval stating whether the requirements of prediction accuracy is met at a given time $t_{\lambda}$ : 

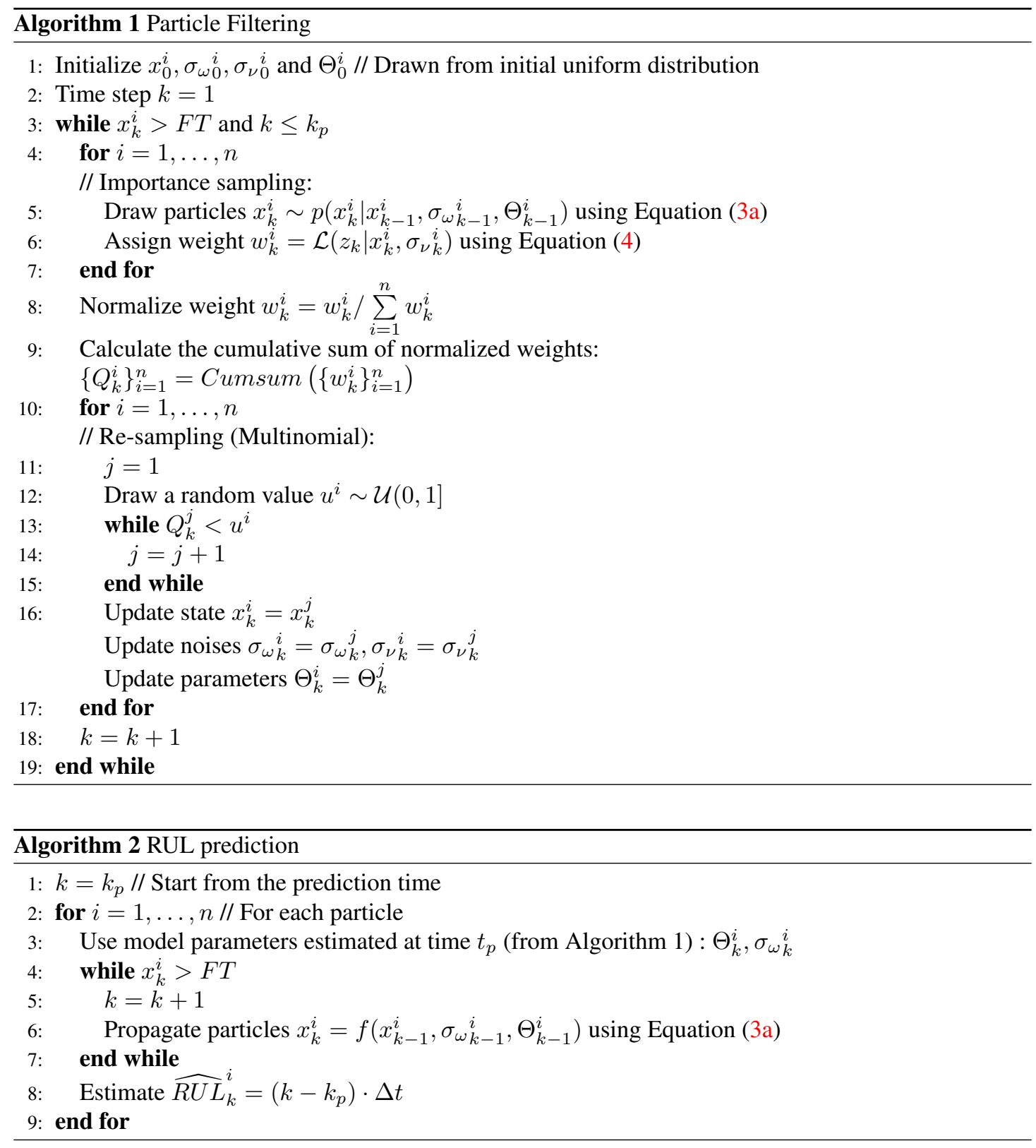

$$
(1-\alpha) \cdot R U L_{\lambda}^{*} \leq \widehat{R U L}_{\lambda} \leq(1+\alpha) \cdot R U L_{\lambda}^{*}
$$




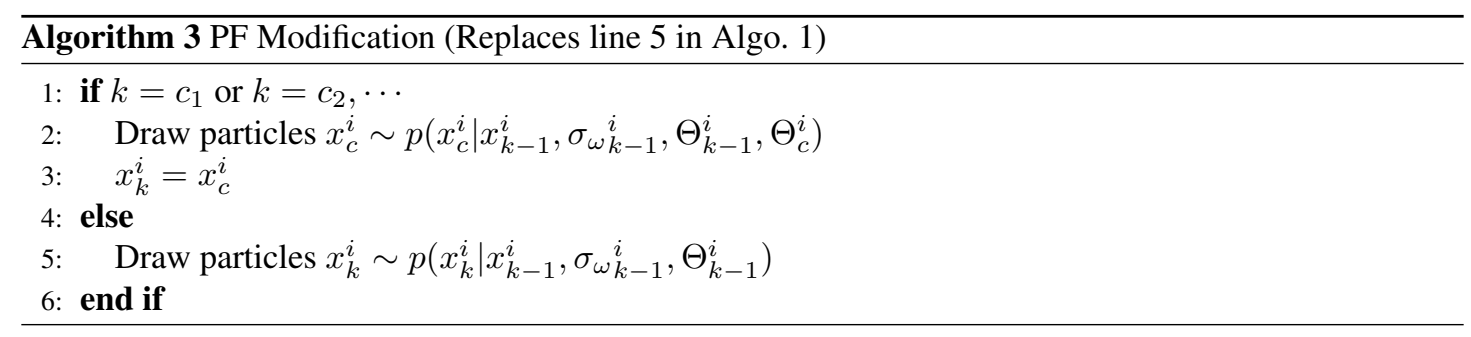

The $\alpha-\lambda$ Accuracy is the probability of this condition being met, higher values correspond to better performance.

Precision The precision $\operatorname{Prc}_{\lambda}$ computes the relative width of the prediction interval, which is defined by:

$$
P r c_{\lambda}=\frac{\widehat{R U L}_{\lambda}^{C I^{+}}-\widehat{R U L}_{\lambda}^{C I^{-}}}{R U L_{\lambda}^{*}}
$$

where $\widehat{R U L}_{\lambda}^{C I^{+}}$and $\widehat{R U L}_{\lambda}^{C I^{-}}$are the upper and lower bounds of the Confidence Interval (CI) of the predicted RULs distribution (e.g. $\mathrm{CI}=50 \%$ ) while $R U L_{\lambda}^{*}$ is the corresponding true RUL. Smaller values of $\operatorname{Prc}_{\lambda}$ indicate more precise predictions.

Coverage The Coverage $C v g_{\lambda}$ is a binary index which considers whether the true RUL lies within the RUL prediction interval at time index $\lambda$ for each trajectory:

$$
C v g_{\lambda}=\widehat{R U L}_{\lambda}^{C I^{-}} \leq R U L_{\lambda}^{*} \leq \widehat{R U L}_{\lambda}^{C I^{+}}
$$

The value of $C v g$ close to $C I$ indicates a good representation of the uncertainty (Baraldi et al. 2013).

\section{Degradation Models}

Choosing a relevant degradation model to build the state transition model of the particle filter is a key step to reach good performance for the PF-based prognostic procedure. In the present case, two deterioration models are required: one for the main degradation trend of the stack voltage, and one for the recovery phenomena. This section presents the used degradation models and details how the stack characterization information is integrated in the model of the recovery phenomena.

\section{Degradation Trend Models}

By degradation models, we refer to empirical models for degradation trends; linear, polynomial, exponential and logarithm models, etc. are commonly used in prognostics (Jouin et al. 2013). By comparing the RUL prediction errors reported in the literature, the 2 nd order polynomial decreasing model proposed in (Kimotho et al. 2014) is chosen for the main degradation trend:

$$
x_{k}=\alpha \cdot\left(t_{k}-t_{k-1}\right)^{2}+\beta \cdot\left(t_{k}-t_{k-1}\right)+x_{k-1}
$$


where $x_{k}$ is the current state, $\alpha$ and $\beta$ are the polynomial degradation model parameters, $t_{k}$ and $t_{k-1}$ are the current time step and previous time step, respectively.

Several models have been tested to adapt the recovery trends, see Table 1. $x_{c}$ is the state including recovery phenomena. In Model $1, \alpha_{0}$ is the average recovery amplitude of power increase after each characterization. In Model 2, the model parameters fit the shape of the recovery phenomena with two parameters: the recovery amplitude $\alpha_{1}$ and its subsequent decreasing trend $\beta_{1}$.

Table 1. Trend Models

\begin{tabular}{cl}
\hline$N^{0}$ & Model \\
\hline classical & $x_{k}$ \\
1 & $x_{c}=x_{k}+\alpha_{0}$ \\
2 & $x_{c}=x_{k}+\alpha_{1} \cdot \exp \left(\beta_{1} \cdot\left(t_{k}-t_{k-1}\right)\right)$ \\
\hline
\end{tabular}

The drawback of Model 2 is that the amplitude of the recovery phenomena changes with the SOH of the stack and tends to higher peaks with time. For that reason, the model is improved in the next section by linking the parameters with the $\mathrm{SOH}$ of the stack.

\section{Recovery Phenomena Model from EIS}

The first stage is to determine which parameter available from EIS results is the most representative of the SOH degradation. The identification results issued from (Kim et al. 2015) show that only the internal resistances have to be taken into account because the other parameters (such as the capacitance $C_{1}$ and $C_{2}$, the inductance $L_{1}$ and $L_{2}$ ) do not exhibit significant changes due to degradation. Besides the polarization resistance is calculated as:

$$
R_{p o l}=\frac{\left(R_{1}+R_{2}\right) \cdot R_{3}}{R_{1}+R_{2}+R_{3}}+R_{e l}
$$

The results of the parameter identification on FC1 and FC2 measurements (Gouriveau et al. 2014), are listed in Table 2.

Table 2. ECM parameters identification.

\begin{tabular}{rcrc}
\hline \multicolumn{2}{c}{ FC1 } & \multicolumn{2}{c}{$\mathrm{FC} 2$} \\
\hline $\mathrm{t}(\mathrm{h})$ & $R_{\text {pol }}(m \Omega)$ & $\mathrm{t}(\mathrm{h})$ & $R_{\text {pol }}(m \Omega)$ \\
\hline 48 & 14.8 & 35 & 15.2 \\
185 & 15.4 & 182 & 15.7 \\
348 & 15.5 & 343 & 16.2 \\
515 & 15.9 & 515 & 17.2 \\
658 & 16.2 & 661 & 16.9 \\
823 & 16.5 & 830 & 17.4 \\
991 & 16.1 & 1016 & 18.6 \\
\hline
\end{tabular}

It can be seen that the resistances generally increase in time, which enables the feasibility of using $R_{p o l}$ as aging indicator. For each characterization time, the polarization resistance can be estimated from 
the polarization curve. Figure 6 pinpoints the correlation between $R_{p o l}$ and the stack power degradation for FC1.

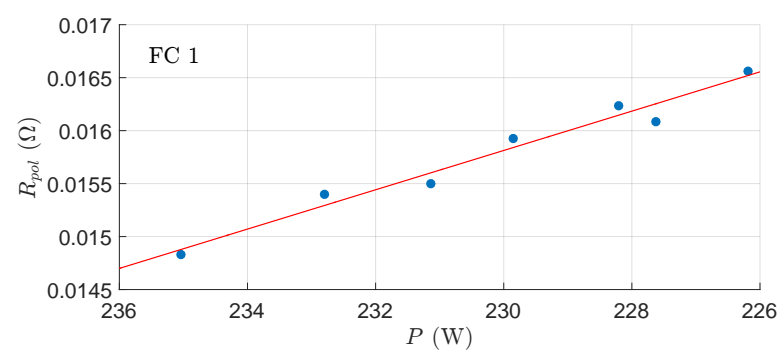

Figure 6. $R_{p o l}$ as a function of the decreasing stack power for FC1.

The R-squared measure of goodness-of-fit of the linear regression is 0.966 for FC1 and 0.969 for FC2, which shows that these data follow a strong linear function for both stacks. The stack power value drops as the resistance increases.

The challenge is now to find relevant relations between $\alpha_{1}$ and $R_{p o l}, \beta_{1}$ and $R_{p o l}$. The variable parameter $R_{p o l}$ can thus be included in a new model (Model 3) as:

$$
x_{c}=x_{k}+\alpha_{2}\left(R_{p o l}\right) \cdot \exp \left(\beta_{2}\left(R_{p o l}\right) \cdot \Delta t\right)
$$

As the fuel cell ages, the recovery reversible amplitude parameter $\alpha_{1}$ and trend parameter $\beta_{1}$ become greater, and so does $R_{p o l}$ as shown in Figure 6. The idea is thus to model this recovery amplitude and this trend parameter as a function of $R_{p o l}$ and we choose to use $R_{p o l}$ as a correcting factor on $\alpha_{1}$ and $\beta_{1}$. We then set:

$$
\begin{aligned}
& \alpha_{2}\left(R_{\text {pol }}\right)=\alpha_{1} \cdot \frac{R_{\text {pol }}(k)}{R_{\text {pol }}(1)} \\
& \beta_{2}\left(R_{\text {pol }}\right)=\beta_{1} \cdot \frac{R_{p o l}(k)}{R_{p o l}(1)}
\end{aligned}
$$

where $R_{p o l}(1)$ is the value of $R_{p o l}$ at $k=1$.

Making the coefficients of the model in Equation (11) dependent on $R_{\text {pol }}$, and not only time-dependent, allows a better adaptation of the recovery model to the internal state of the FC, and consequently a better prognosis, as shown in the next section.

\section{RUL Prediction Results}

PF Settings

The estimation performed by PF (e.g. in Figure 7), is realized with $N=5000$ particles. Since there are no available prior information, it is assumed that the initial distributions of the system state $x$ and the unknown parameters $\Theta$ are uniformly distributed between their lower and upper bounds: 


$$
\begin{aligned}
x_{0} & \sim \mathcal{U}\left(x_{0}^{-}, x_{0}^{+}\right) \\
\Theta_{0} & \sim \mathcal{U}\left(\Theta_{0}^{-}, \Theta_{0}^{+}\right)
\end{aligned}
$$

The bounds of $x_{0}$ are set to $\pm 5 \mathrm{~W}$ around FC1 initial power (represented by the first measurement) and the measurement noise variance $\sigma_{\nu 0}^{2} \in(0,5)$ is set accordingly to ensure a good estimation of the noisy measurement. The value for process noise variance is found through successive tuning as $\sigma_{\omega}^{2} \in(0,0.04)$, to obtain a desirably smooth estimation. It is necessary to note that, for the measurement function in Eq.(3a), it is assumed that $y_{k}$ is the same as the output power including measurement noise $\nu_{k}$. In the PF framework, particles will be updated by being propagated through the state transition model in Eq.(9). When the scheduled characterizations take place, they are updated according to the models described in Table 1 and Eq. 11.

\section{Stationary Regime (FC1)}

The stack power degradation of FC1 over 1000 hours is estimated by PF for all the models. The RUL of a PEMFC stack is predicted regarding its output power Failure Threshold (FT). The FT of the stack is defined as a given portion of its initial output power (e.g. $96 \%$ of its power at $t=0 \mathrm{~h}$ ).

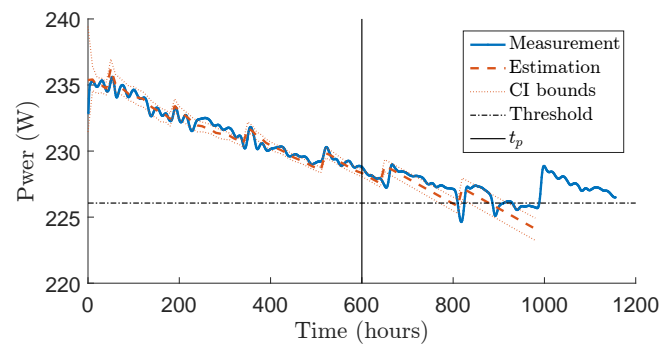

Figure 7. Degradation estimation for FC1 (Model 3).

Figure 7 shows an example of the estimation of degradation path at prediction time $t_{\lambda}=600$ hours for Model 3. One can see the recovery phenomena have been adapted both in learning and prediction phases. The predicted RUL with its uncertainty is illustrated in Figure 8. The bi-modal PDF of the predicted RUL is due to the specific behavior of the considered fuel cell around time 800 hours : it can be explained by the fact that there is a high amplitude recovery phenomena just at the same time when the deterioration is about to cross the failure threshold, see Figure 7. Taking into account the uncertainty, this could lead either to a failure around $800 \mathrm{~h}$ or, in the cases where the propagated deterioration does cross the threshold at 800h, to a later failure around 900h; hence the bi-modal PDF that integrates these two possible scenarios. With $80 \% C I$, the predicted RUL at $t_{\lambda}=600$ hours is within $[170 ; 330]$ whereas the true RUL is 210 hours.

The RUL predictions are then applied with time index $t_{\lambda}$ from 200 hours to the End of Life (EOL) every 20 hours to evaluate the average performance of each model. The boxplot in Figure 9 shows the predicted RUL uncertainties with $C I=50 \%$ and accuracy bounds $\alpha=0.2$. 


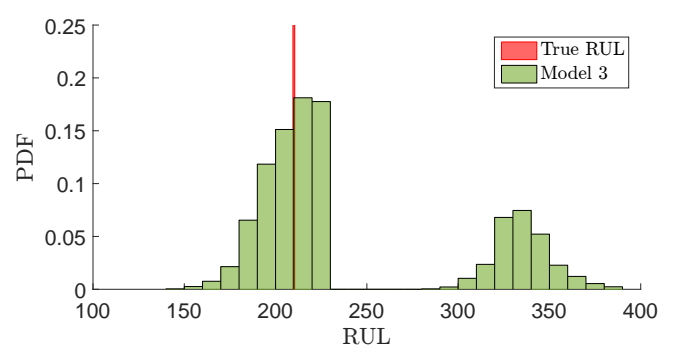

Figure 8. Histogram of the predicted RUL for FC1 at 600 hours (Model 3).

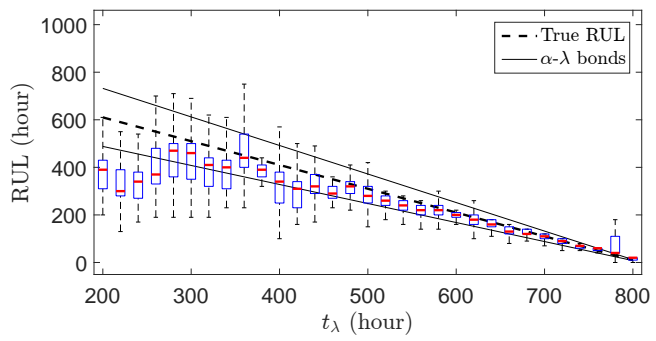

Figure 9. Model 3 RUL predictions with uncertainties for FC1 ( $\alpha=0.2$ and $C I=50 \%)$.

The performances evaluated by all metrics for FC1 are compared in Table 3. Model 3 shows better performances on $\alpha-\lambda$ Accuracy and Acc indexes which implies that this model gives more accurate predictions than other models. Such a model also has better performances on Prc (the lowest value) and $C v g$ (the closest to $C I=80 \%$ ), which indicates that it provides more precise RUL predictions while maintaining a better coverage performance than other models.

Table 3. Prognostic performance for FC1 $(\alpha=0.2, C I=80 \%)$.

\begin{tabular}{ccccc}
\hline & Classical & Model 1 & Model 2 & Model 3 \\
\hline$\alpha-\lambda$ & 0.645 & 0.548 & 0.677 & $\mathbf{0 . 7 7 4}$ \\
$A c c$ & 0.675 & 0.793 & 0.810 & $\mathbf{0 . 8 5 6}$ \\
$P r c$ & 0.894 & 0.853 & 0.731 & $\mathbf{0 . 6 8 1}$ \\
$C v g$ & 0.742 & 0.581 & 0.6129 & $\mathbf{0 . 7 7 4}$ \\
\hline
\end{tabular}

Figure 10 shows the RUL predictions with $\alpha$ bounds of all models. It can be seen that the results of Model 3 are better suited within the bounds than the others.

\section{Dynamic Current Regime (FC2)}

The same algorithm is applied to the other stack FC2. In Figure 11, the classical model gives a realistic RUL prediction, but this prediction may not be robust to other experiments, as the characterization 


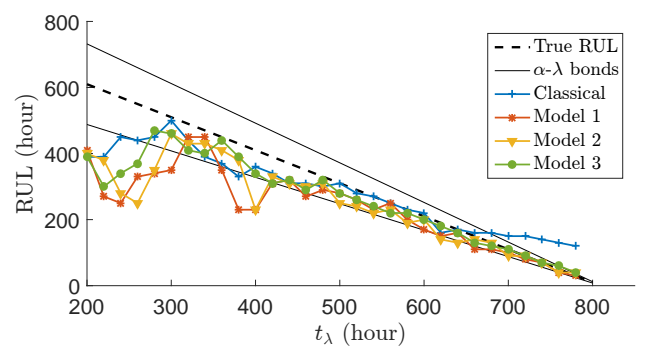

Figure 10. RUL predictions with different models for FC1 $(\alpha=0.2)$.

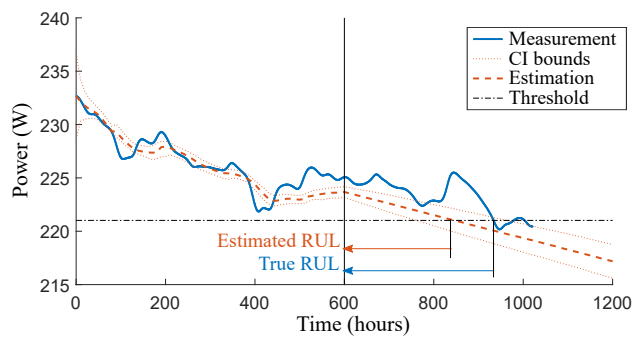

Figure 11. Degradation estimation for FC2 (Classical PF).

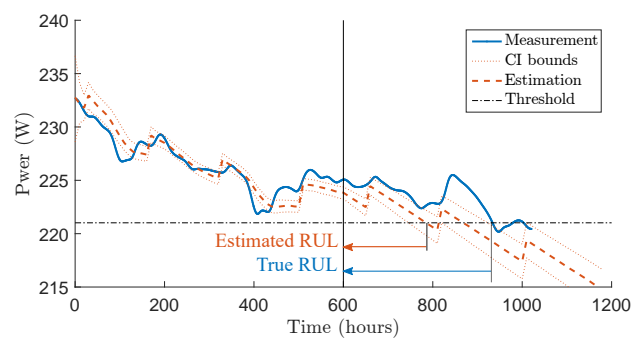

Figure 12. Degradation estimation for FC2 (Model 1).

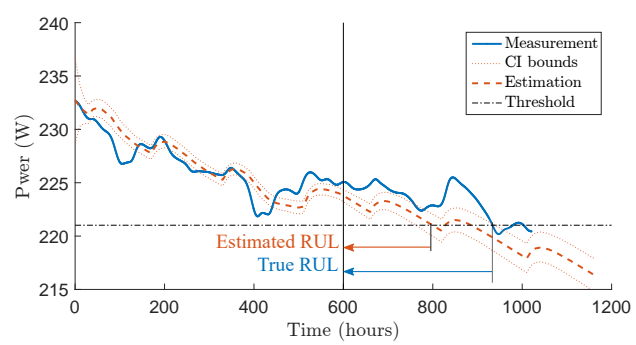

Figure 13. Degradation estimation for FC2 (Model 2). 


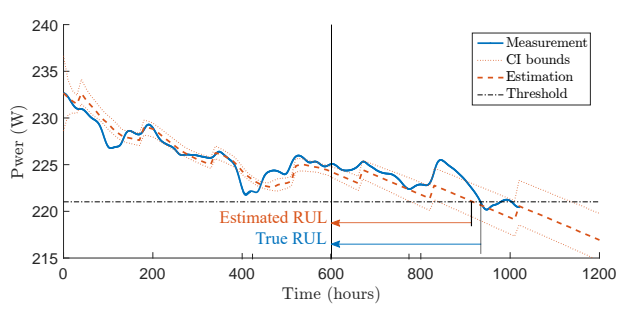

Figure 14. Degradation estimation for FC2 (Model 3).

times are not taken into account. For Model 1 and Model 2, the characterization times are taken into consideration, but the magnitude and the rate are estimated during data training. Thus, the prediction RUL is too short (Figure 12 and Figure 13). For Model 3, taking into account the polarization resistance allows adapting the magnitude and the degradation rate (Figure 14). The RUL prediction is thus more accurate than the other models. However, it can be seen that the peak at time $850 \mathrm{~h}$ cannot be predicted without additional information on this event.

An example of predicted RUL PDFs $\left(e . g\right.$. at $\left.t_{\lambda}=600 h\right)$ by all models is shown in Figure 15. One can see that the RUL PDFs are thinner for the model 3. The boxplot in Figure 16 shows the predicted RUL uncertainties of model 3. This figure highlights that the dramatic drop between $t=400 \mathrm{~h}$ and $t=500 \mathrm{~h}$ induces difficulties in the RUL prediction. Figure 17 shows the RUL predictions with $\alpha$ bounds of all models and classical PF. One can see that Model 3 gives the best predictions after $t=500 h$.

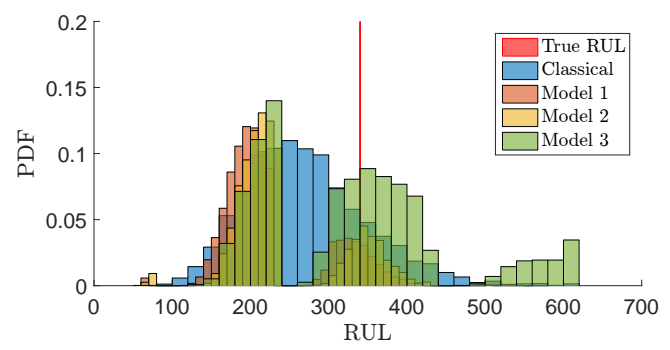

Figure 15. Predicted RUL for FC2 at 600 hours.

Table 4 reports all performance metrics obtained when using all models for FC2. It can be noticed that the performances on FC2 are globally worse when compared with those on FC1. The classical PF provides in this case more precise predictions but lacks accuracy. Model 3 shows better performances on $\alpha-\lambda$ Accuracy, Acc and Cvg indexes. The poor performance on Prc index for Model 3 is due to the fact that the predicted RUL distribution does not converge as fast as with the classical PF because of the adaption to the recovery phenomena. 


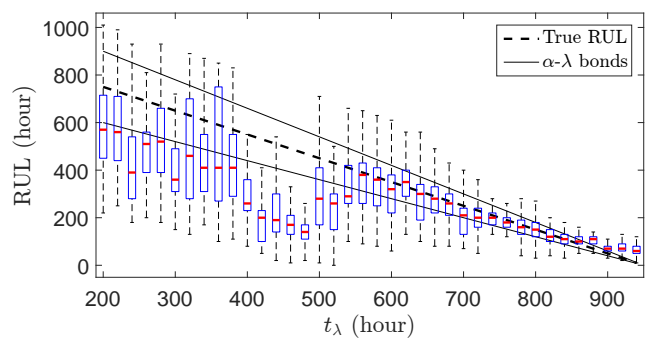

Figure 16. Model $3 \mathrm{RUL}$ predictions with uncertainties for FC2 ( $\alpha=0.2$ and $C I=50 \%)$.

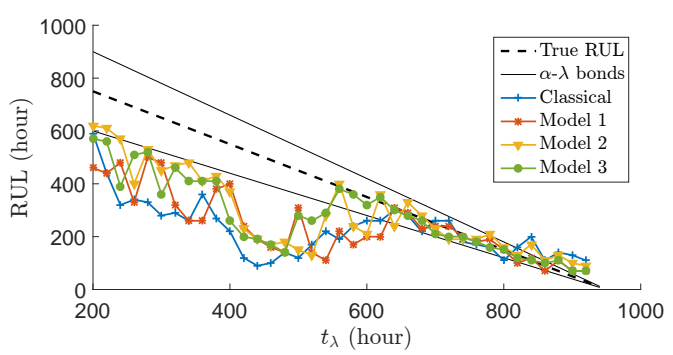

Figure 17. RUL predictions with different models for FC2 $(\alpha=0.2)$.

Table 4. Prognostic performance for FC2 $(\alpha=0.2, C I=80 \%)$.

\begin{tabular}{ccccc}
\hline & Classical & Model 1 & Model 2 & Model 3 \\
\hline$\alpha-\lambda$ & 0.237 & 0.263 & 0.342 & $\mathbf{0 . 4 2 1}$ \\
$A c c$ & 0.485 & 0.632 & 0.633 & $\mathbf{0 . 7 1 8}$ \\
Prc & $\mathbf{0 . 6 9 5}$ & 0.765 & 0.932 & 0.755 \\
Cvg & 0.342 & 0.553 & 0.632 & $\mathbf{0 . 7 8 9}$ \\
\hline
\end{tabular}

\section{Conclusion}

The purpose of this study was to improve the prognostic quality of the empirical power degradation model by handling the impact of PEMFC stacks' recovery behavior. The parameter of polarization resistance estimated from EIS helped to improve the RUL prediction accuracy. The proposed model gives the best performance among all the tested models, especially for long-term predictions. This study brings the idea of integrating SOH characterization into RUL prediction, which leads to a better performance of RUL predictions. Even if the results obtained on the set of real data considered in the paper are promising, the proposed approach still has to be tested and validated in a broader range of data which for sure will raise new challenges. In future work, other available information regarding the $\mathrm{SOH}$ characterization and their interest for RUL prediction will also be explored. 


\section{Acknowledgements}

This work has been partly supported by Labex ACTION program (ANR-11-LABX-0001-01) and InnoEnergy PhD School program.

\section{References}

Arulampalam MS, Maskell S, Gordon N and Clapp T (2002) A tutorial on particle filters for online nonlinear/nonGaussian Bayesian tracking. IEEE Trans. Signal Process. 50(2): 174-188. DOI:10.1109/78.978374.

Baraldi P, Compare M, Sauco S and Zio E (2013) Ensemble neural network-based particle filtering for prognostics. Mech. Syst. Signal Process. 41(1-2): 288-300. DOI:10.1016/j.ymssp.2013.07.010.

Bressel M, Hilairet M, Hissel D and Ould Bouamama B (2016) Remaining Useful Life Prediction and Uncertainty Quantification of Proton Exchange Membrane Fuel Cell Under Variable Load. IEEE Trans. Ind. Electron. 63(4): $1-1$.

Dubau L, Castanheira L, Maillard F, Chatenet M, Lottin O, Maranzana G, Dillet J, Lamibrac A, Perrin JC, Moukheiber E, Elkaddouri A, De Moor G, Bas C, Flandin L and Caqué N (2014) A review of PEM fuel cell durability: Materials degradation, local heterogeneities of aging and possible mitigation strategies. Wiley Interdisciplinary Reviews: Energy and Environment 3(6): 540-560. DOI:10.1002/wene.113.

Gouriveau R, Hilairet M, Hissel D, Jemeï S, Jouin M, Lechartier E, Morando S, Pahon E, Péra MC and Zerhouni N (2014) IEEE PHM 2014 Data Challenge - Outline, Experiments, Scoring of results, Winners.

Javed K, Gouriveau R, Zerhouni N and Hissel D (2016) Prognostics of Proton Exchange Membrane Fuel Cells stack using an ensemble of constraints based connectionist networks. J. Power Sources 324: 745-757. DOI: 10.1016/j.jpowsour.2016.05.092.

Jha MS, Dauphin-Tanguy G and Ould-Bouamama B (2016) Particle filter based hybrid prognostics of proton exchange membranefuel cell in bond graph framework. Mech. Syst. Signal Process. 75: 301-329. DOI: 10.1016/j.ymssp.2016.01.010.

Jouin M, Gouriveau R, Hissel D and Member S (2016a) Joint Particle Filters Prognostics for Proton Exchange Membrane Fuel Cell Power Prediction at Constant Current Solicitation. Ieee Trans. Reliab. 65(1): 336-349. DOI:10.1109/TR.2015.2454499.

Jouin M, Gouriveau R, Hissel D, Péra MC and Zerhouni N (2013) Prognostics and Health Management of PEMFC - State of the art and remaining challenges. Int. J. Hydrogen Energy 38(35): 15307-15317. DOI: 10.1016/j.ijhydene.2013.09.051.

Jouin M, Gouriveau R, Hissel D, Péra MC and Zerhouni N (2016b) Particle filter-based prognostics: Review, discussion and perspectives. Mech. Syst. Signal Process. DOI:10.1016/j.ymssp.2015.11.008.

Kim T, Kim H, Ha J, Kim K, Youn J, Jung J and Youn BD (2015) A degenerated equivalent circuit model and hybrid prediction for state-of-health (SOH) of PEM fuel cell. In: 2014 Int. Conf. Progn. Heal. Manag. PHM 2014. ISBN 978-1-4799-4943-4, pp. 1-7. DOI:10.1109/ICPHM.2014.7036407.

Kimotho JK, Meyer T and Sextro W (2014) PEM fuel cell prognostics using particle filter with model parameter adaptation. In: 2014 Int. Conf. Progn. Heal. Manag. ISBN 978-1-4799-4943-4, pp. 1-6. DOI:10.1109/ICPHM. 2014.7036406.

Lechartier E, Laffly E, Péra MC, Gouriveau R, Hissel D and Zerhouni N (2015) Proton exchange membrane fuel cell behavioral model suitable for prognostics. Int. J. Hydrogen Energy 40(26): 8384-8397. DOI: 10.1016/j.ijhydene.2015.04.099. 
Li T, Bolic M and Djuric PM (2015) Resampling methods for particle filtering: Classification, implementation, and strategies. IEEE Signal Processing Magazine 32(3): 70-86. DOI:10.1109/MSP.2014.2330626.

Morando S, Jemei S, Hissel D, Gouriveau R and Zerhouni N (2017) ANOVA method applied to proton exchange membrane fuel cell ageing forecasting using an echo state network. Math. Comput. Simul. 131: 283-294. DOI: 10.1016/j.matcom.2015.06.009.

Rigamonti M, Baraldi P, Zio E, Astigarraga D and Galarza A (2016) Particle Filter-Based Prognostics for an Electrolytic Capacitor Working in Variable Operating Conditions. IEEE Trans. Power Electron. 31(2): 15671575. DOI:10.1109/TPEL.2015.2418198.

Saxena A, Celaya J, Saha B, Saha S and Goebel K (2010) Metrics for Offline Evaluation of Prognostic Performance. Int. J. Progn. Heal. Manag. 1(1): 1-20.

Vachtsevanos G, Lewis FL, Roemer M, Hess A and Wu B (2006) Intelligent Fault Diagnosis and Prognosis for Engineering Systems. Hoboken: John Wiley \& Sons. ISBN 978-0-471-72999-0.

Zhang D, Cadet C, Yousfi-Steiner N and Bérenguer C (2016) Some Improvements of Particle Filtering Based Prognosis for PEM Fuel Cells. In: IFAC-PapersOnLine, volume 49. pp. 162-167. DOI:10.1016/j.ifacol.2016. 11.028 . 